This item was submitted to Loughborough's Research Repository by the author.

Items in Figshare are protected by copyright, with all rights reserved, unless otherwise indicated.

\title{
Interplay of size and Landau quantizations in the de Haas-van Alphen oscillations of metallic nanowires
}

PLEASE CITE THE PUBLISHED VERSION

PUBLISHER

(C) American Physical Society

\section{VERSION}

VoR (Version of Record)

\section{LICENCE}

CC BY-NC-ND 4.0

\section{REPOSITORY RECORD}

Alexandrov, A.S., V.V. Kabanov, and I.O. Thomas. 2019. "Interplay of Size and Landau Quantizations in the De Haas-van Alphen Oscillations of Metallic Nanowires". figshare. https://hdl.handle.net/2134/4248. 
This item was submitted to Loughborough's Institutional Repository (https://dspace.lboro.ac.uk/) by the author and is made available under the following Creative Commons Licence conditions.

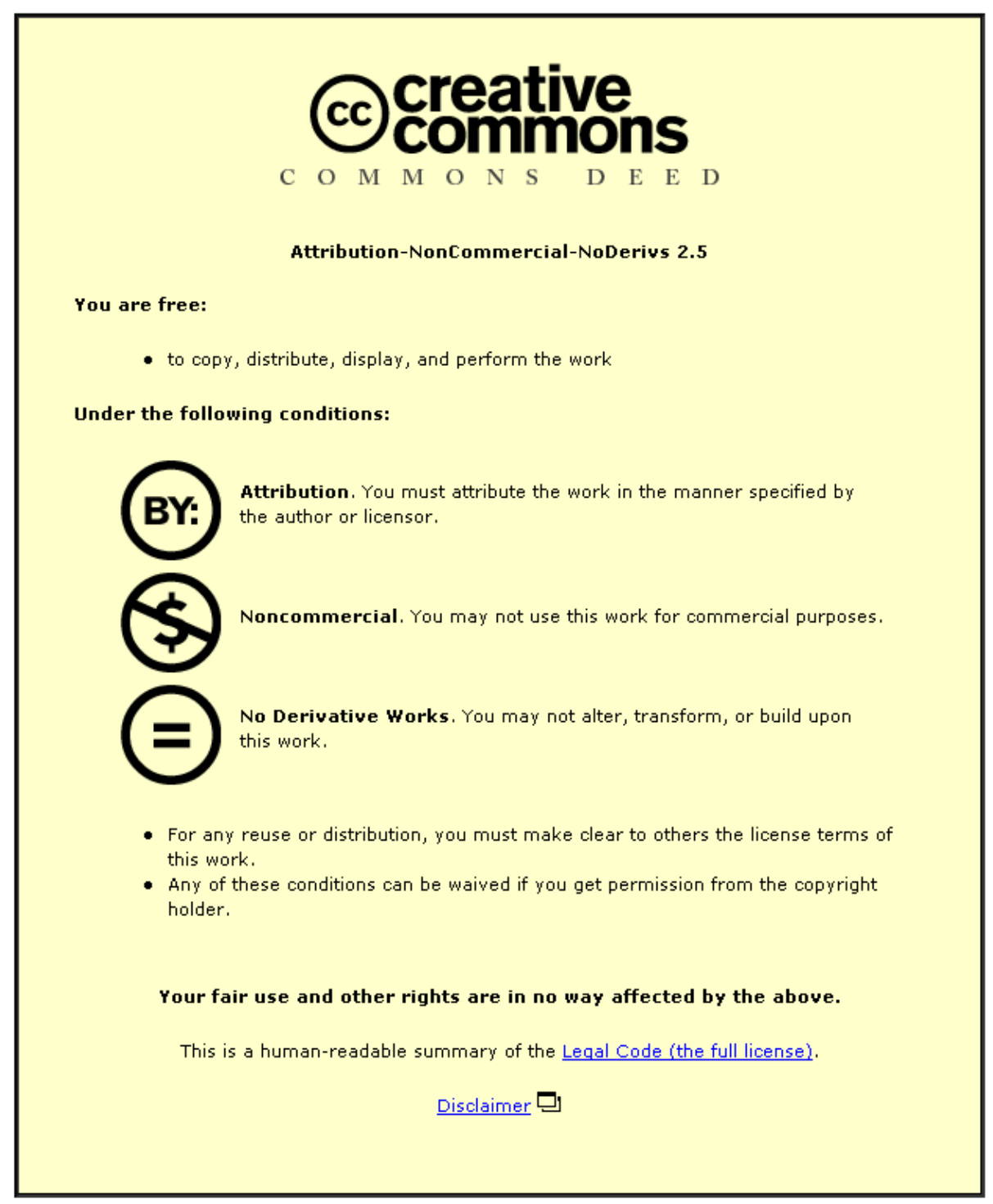

For the full text of this licence, please go to: http://creativecommons.org/licenses/by-nc-nd/2.5/ 


\title{
Interplay of size and Landau quantizations in the de Haas-van Alphen oscillations of metallic nanowires
}

\author{
A. S. Alexandrov, ${ }^{1}$ V. V. Kabanov, ${ }^{2}$ and I. O. Thomas ${ }^{1}$ \\ ${ }^{1}$ Department of Physics, Loughborough University, Loughborough, Leicestershire LE11 3TU, United Kingdom \\ ${ }^{2}$ Josef Stefan Institute, 1001 Ljubljana, Slovenia \\ (Received 8 May 2007; revised manuscript received 27 July 2007; published 18 October 2007)
}

\begin{abstract}
We examine the interplay between size quantization and Landau quantization in the de Haas-van Alphen oscillations of clean metallic nanowires in a longitudinal magnetic field for "hard" boundary conditions, i.e., those of an infinite round well, as opposed to the "soft" parabolically confined boundary conditions previously treated in Alexandrov and Kabanov (AK) [Phys. Rev. Lett. 95, 076601 (2005)]. We find that there exist two fundamental frequencies as opposed to the one found in bulk systems and the three frequencies found by AK with soft boundary counditions. In addition, we find that the additional "magic resonances" of AK may also be observed in the infinite well case, though they are now damped. We also compare the numerically generated energy spectrum of the infinite well potential with that of our analytic approximation, and compare calculations of the oscillatory portions of the thermodynamic quantities for both models.
\end{abstract}

DOI: 10.1103/PhysRevB.76.155417

PACS number(s): 73.63.Nm, 75.75.+a

\section{INTRODUCTION}

Since their discovery in $1930,{ }^{1}$ and subsequent explanation by Onsager ${ }^{2}$ and Lifshitz and Kosevitch ${ }^{3}$ in terms of the interplay between the Landau quantization and the Fermi surface, the de Haas-van Alphen (DHVA) oscillations of the thermodynamic potential, magnetization, and related quantities have acquired a long history of use as a method of probing the structure of the Fermi surface of metals at low temperatures and large magnetic fields. (A summary of the first 38 years of study in this field may be found in Gold. ${ }^{4}$ More contemporary reviews of the area may be found in Schoenberg, ${ }^{5}$ Singleton ${ }^{6}$ and Kartsovnik, ${ }^{7}$ with the latter two focusing on applications to quasi-two-dimensional organic compounds.)

The question of boundary conditions and how confinement affects the behavior of electrons in longitudinal magnetic fields (and hence the DHVA effect) has long been a question of interest to the condensed matter community (see, for example, Fock, ${ }^{8}$ Landau, ${ }^{9}$ Darwin, ${ }^{10}$ and also the discussion in Dingle's classic paper ${ }^{11}$ on the effects of boundary conditions on the density of states in large systems). This issue is of obvious importance in the study of nanowires, where the system of interest is confined within a narrow, cylindrical potential, which in this case is difficult to treat analytically owing to the difficulties in approximating the eigenvalues of the Schrödinger equation given by the zeros of the confluent hypergeometric function. However, in the weak field limit, the eigenvalues are well approximated by the zeros of the Bessel function, and so Dingle ${ }^{12}$ was able to calculate the effect of such a confinement in a weak magnetic field through treating the latter as a small perturbation to the energy levels and showing that at low fields, one should expect to observe oscillations that are primarily due to the passage of the size quantized energy levels above the Fermi energy. Bogachek and Gogadze ${ }^{13}$ calculated the contribution to the thermodynamic potential of those electrons near the surface of the wire that are on a "grazing" trajectory with respect to the surface, and argued that this is, in fact, a more dominant contribution to the thermodynamic potential than the Dingle-type oscillations, as the latter are suppressed by specular reflection under normal circumstances. The contribution of these surface levels corresponds to that induced by the Aharonov-Bohm effect. This effect was observed, along with others ascribed to Dingle-type oscillations by Brandt et al. ${ }^{14}$ in a study of the magnetoresistance of bismuth wires of micrometer thickness. Further confirmations of the presence of the low field Aharonov-Bohm effect before the onset of Shubnikov-de Haas oscillations in the magnetoresistance come from the study of progressively narrower $\mathrm{Bi}$ nanowires, ${ }^{15}$ as well as of carbon nanotubes. ${ }^{16,17}$

It is likely that at higher fields for wires of very narrow radius, we might expect additional effects of the interplay between size quantization and Landau quantization to become apparent. There is a need for theoretical treatments of this region, and in what follows we make an attempt at doing so outside of an idealized one-dimensional limit, such as that used in Sineavsky et al. ${ }^{18}$

Alexandrov and Kabanov (AK) ${ }^{19}$ have recently examined the DHVA oscillations present in a system of electrons confined within a parabolic confining potential (the FockDarwin or confinement model ${ }^{8,10}$ ). They have found that there exist three characteristic frequencies of DHVA oscillations, and also that the thermodynamic potential displays "magic resonances" at certain values of the field where the amplitude is greatly enhanced. In a brief note at the end of this Introduction, we demonstrate that despite being due to a divergent cotangent function in our expression for the oscillatory thermodynamic potential $\widetilde{\Omega}$, the behavior of the thermodynamic potential is still analytically well defined-this is important if the AK result is to be properly understood.

Our paper focuses on a system of free electrons confined within a cylindrical infinite well potential and subject to a longitudinal magnetic field. Using a well known asymptotic description of the wave function of such a system, we derive a nonlinear energy spectrum and confirm it by deriving a similar result through a semiclassical approximation. By linearizing these spectra near the Fermi energy, we find that we 
acquire expressions for the energy similar to that of the Fock-Darwin model, and so we may motivate an improvement to the asymptotic and semiclassical models that brings them closer to the energy spectrum at larger values of the field than was previously possible. The oscillations in the thermodynamic potential are calculated, and we find not only that the additional characteristic frequencies of the system are modified, but also that the position of the magic resonances has altered and that they are somewhat damped. Numerical plots of the thermodynamic quantities calculated are given for various values of the field.

Finally, we examine how well the modified asymptotic approximates the exact spectrum of the problem. We find that it is a reasonable approximation for low values of the magnetic quantum number $m$ at large $\omega_{c}=e B / \mathrm{m}^{*} c$; however, it is inaccurate for large values of $m$, reflecting orbits that are closer to the edge of the wire. This is likely because the asymptotic that we are using is not valid in that limit (similarly, the semiclassical approximation breaks down here since it is too close to a turning point of the classical electron trajectories). However, the actual boundary conditions of the system are most likely intermediate between those of the infinite cylindrical well and those of the Fock-Darwin confinement model, and so it is perhaps reasonable to suspect that the breakdown of the approximation, which is likely due to the "hardness" of the boundary conditions in the infinite well case, should not unduly restrict the application of the nonlinear spectrum to real systems.

In what follows, we assume that the system of interest is a long, clean metallic nanowire in a longitudinal magnetic field $\mathbf{B}$ that is parallel to the direction of the wire (taken to be the $z$ axis of a cylindrical coordinate system). We take the electron mean free path, $l=v_{F} \tau$ to be comparable to or larger than the radius of the wire $R$, but not the length $L$. We also assume that the electron wavelength near the Fermi level is very small in these metallic nanowires, such that $L \gg l \leqq R$ $\gg 2 \pi \hbar /\left(m^{*} v_{F}\right)$; here and previously, $v_{F}$ is the Fermi velocity and $m^{*}$ is the band mass in the bulk metal.

Henceforth, we also take $\hbar=c=m^{*}=L=1$.

\section{A. On the divergence of the magic resonances in the Alexandrov-Kabamov result}

The $\mathrm{AK}^{19}$ result describes the DHVA oscillations of a three-dimensional electron exposed to a magnetic field within a two-dimensional parabolic confining potential. ${ }^{8,10}$ This system is an isotropic planar harmonic oscillator whose frequency in the absence of a magnetic field is taken to be $\omega_{0}$. Due to the effects of confinement, the DHVA oscillations of the system aquire three characteristic frequencies of oscillation: $2 \omega, \omega^{+}$, and $\omega^{-}$, where $\omega^{2}=\omega_{0}^{2}+\omega_{c}^{2} / 4$ and $\omega^{ \pm}$ $=\omega \pm \omega_{c} / 2$. The amplitudes are given by

$$
A_{r}(x, y)=\frac{T(2 x)^{1 / 2}}{2 \pi r^{3 / 2} \sinh \left[\pi^{2} T r /(x)\right]} \cot \left(\frac{\pi r y}{2 x}\right),
$$

where $x=\omega$ and $y=\omega^{ \pm}$for the the amplitudes of the oscillations with frequency $2 \omega$, and $x=\omega^{ \pm} / 2$ and $y=2 \omega$ for those with frequency $\omega^{ \pm}$. As has been previously noted, these amplitudes [Eq. (1)] contain a cotangent function which gives rise to magic resonances for certain values of the field where the condition $2 \omega /\left(\omega^{ \pm}\right)=(q+2) / r$ is satisfied. If this is the case, then the cotangent becomes infinite provided that $q$ is an integer.

However, despite what one might naively assume, this apparent divergence is, in fact, analytically well defined. Let us examine the following harmonics: $r=1, q=1$ and $r=3, q$ $=7$. From our condition for the divergence of the cotangent, we have $\omega=\frac{3}{2} \omega_{c}$ and, hence, $\omega^{-}=\frac{2}{3} \omega$. Let us set $\omega=1$, and then set $\omega^{-}=\frac{2}{3}(1-\delta)$, where $\delta \rightarrow+0$.

For our $r=1$ harmonic, we shall examine the $A_{1}\left(\frac{\omega^{-}}{2}, 2 \omega\right)$ amplitude, and for our $r=3$ harmonic, we shall examine the $A_{3}\left(\omega, \omega^{-}\right)$amplitude. We acquire

$A_{1}\left(\frac{\omega^{-}}{2}, 2 \omega\right)=A_{1}\left(\frac{1}{3}(1-\delta), 2\right)=\frac{T(2 / 3)^{1 / 2}}{2 \pi \sinh \left[3 \pi^{2} T\right]} \cot \left(\frac{3 \pi}{1-\delta}\right)$

and

$A_{3}\left(\omega, \omega^{-}\right)=A_{3}\left(1, \frac{2}{3}(1-\delta)\right)=\frac{T(2 / 3)^{1 / 2}}{6 \pi \sinh \left[3 \pi^{2} T\right]} \cot [\pi(1-\delta)]$.

Expanding out the cotangent from Eq. (2), we find $\cot [3 \pi /(1-\delta)] \approx 1 /(3 \pi \delta)$, while doing the same for Eq. (3) gives $\cot [\pi(1-\delta)] \approx-1(\pi \delta)$. Substituting these values back into Eq. (2) and (3), respectively, we find that

$$
\begin{aligned}
A_{1}\left(\frac{1}{3}(1-\delta), 2\right) & =-A_{3}\left(1, \frac{2}{3}(1-\delta)\right) \\
& =\frac{T(2 / 3)^{1 / 2}}{6 \pi^{2} \sinh \left[3 \pi^{2} T\right]} \frac{1}{\delta} \\
& \equiv \frac{A}{\delta}
\end{aligned}
$$

We may now write the following, where $\widetilde{\Omega}_{\text {res }}$ is the oscilliatory component of the AK result near the magic resonance:

$$
\begin{aligned}
\widetilde{\Omega}_{r e s}= & A_{3}\left(\omega, \omega^{-}\right) \sin \left(\frac{3 \mu \pi}{\omega}-\frac{3 \pi\left(\omega^{+}+\omega^{-}\right)}{2 \omega}-\frac{\pi}{4}\right) \\
& +A_{1}\left(\frac{\omega^{-}}{2}, 2 \omega\right) \sin \left(\frac{2 \mu \pi}{\omega^{-}}-\frac{3 \pi\left(\omega^{+}+\omega^{-}\right)}{\omega^{-}}-\frac{\pi}{4}\right) \\
= & -\frac{A}{\delta} \sin \left[3 \mu \pi-\frac{3 \pi}{2}\left(\omega+\frac{2}{3}(1-\delta)\right)-\frac{\pi}{4}\right] \\
& +\frac{A}{\delta} \sin \left[\frac{3 \mu \pi}{1-\delta}-\frac{3 \pi}{2(1-\delta)}\left(\omega+\frac{2}{3}(1-\delta)\right)-\frac{\pi}{4}\right] .
\end{aligned}
$$

Defining $C \equiv 3 \mu \pi-\frac{3}{2} \pi\left(\omega^{+}+\frac{2}{3}\right)$ and expanding out the terms in the denominators, we arrive at

$$
\widetilde{\Omega}_{r e s}=A C \cos \left(C-\frac{\pi}{4}\right) .
$$

Note that the singular terms are no longer present, so that the thermodynamic potential in the region of the resonance is now finite. However, it has also been enhanced by a factor of 
$C$, which is of the order of the Fermi energy $\mu \gg 1$, and so remains rather large. We would expect the same analysis to hold true for the other magic resonances.

\section{CYLINDRICAL CONFINEMENT AND THE DE HASS-VAN ALPHEN EFFECT}

The Schrödinger equation in polar coordinates for an electron moving in a longitudinal magnetic field $B$ parallel to the $z$ axis is

$$
-\frac{1}{2}\left[\frac{1}{r} \frac{\partial}{\partial r}\left(r \frac{\partial \psi}{\partial r}\right)+\frac{\partial^{2} \psi}{\partial z^{2}}+\frac{1}{r^{2}} \frac{\partial^{2} \psi}{\partial \phi^{2}}\right]+\frac{i \omega_{c}}{2} \frac{\partial \psi}{\partial \phi}+\frac{\omega_{c}^{2} r}{8} \psi=E \psi .
$$

Following, for example (though note the difference in convention regarding $e$ ), Landau and Lifshitz, ${ }^{20}$ we seek a solution in the form

$$
\psi=\frac{1}{\sqrt{2 \pi}} R(r) e^{i m \phi} e^{i p_{z}},
$$

where $R(r)$, after redefinition in terms of $\xi=\left(\omega_{c} / 2\right) r^{2}$, is the radial function,

$$
R(\xi)=e^{-\xi / 2} \xi^{|m| / 2} M\left[-\left(\beta-\frac{|m|}{2}-\frac{1}{2}\right),|m|+1, \xi\right],
$$

with $\beta=\omega_{c}^{-1}\left[E-\left(p_{z}^{2} / 2\right)\right]+m / 2 . M\left[-\left(\beta-\frac{|m|}{2}-\frac{1}{2}\right),|m|+1, \xi\right]$ is a confluent hypergeometric function. The zeros of this are the eigenvalues of the Schrödinger equation for cylindrically confined electrons. If we write $\beta-\frac{|m|}{2}-\frac{1}{2}$ as $-a$, following the notation of Abramowitz and Stegun, ${ }^{21}$ the energy levels of the particle are given by ${ }^{20}$

$$
E=\frac{k^{2}}{2}+\omega_{c}\left(-a+\frac{|m|-m+1}{2}\right) .
$$

If the wave function is finite everywhere, then $-a$ is an integer, and the eigenfunctions of the equation are the Laguerre polynomials. However, if this is not the case, and it becomes zero at some finite radius $R$, then $-a$ is a positive, real number. In order to proceed analytically in these cases, particularly where $R$ is small, we must therefore use various approximations or numerical methods so as to obtain the energy spectrum.

\section{A. Asymptotic approximation}

For large enough values of $a$, where $a$ is negative, the confluent hypergeometric function $M(a, b, \xi)$ may be approximated by the following asymptotic form: ${ }^{21}$

$$
\begin{aligned}
M(a, b, \xi)= & \Gamma(b) e^{\xi / 2}\left(\frac{1}{2} b \xi-a \xi\right)^{(1-b) / 2} \pi^{-1 / 2} \cos (\sqrt{2 b \xi-4 a \xi} \\
& \left.-\frac{1}{2} b \pi+\frac{1}{4} \pi\right)
\end{aligned}
$$

where (as before) we take $a=-\left(\omega_{c}^{-1}\left[E-p_{z}^{2} / 2\right]+\frac{m}{2}-\frac{|m|}{2}-\frac{1}{2}\right)$, $b=|m|+1$, and $\xi=\omega_{c} R^{2} / 2=\left(\pi / 2 \omega_{s}\right)^{2} \mu \omega_{c}$, with $R$ being the radius of the nanowire and $\omega_{S}=\pi v_{F} / 2 R$. In so choosing the value of $\xi$, we are imposing hard boundary conditions by stating that the wave function must be zero at $R$.

Our approximation has zeros when the following is true:

$$
\sqrt{2 b \xi-4 a \xi}-\frac{1}{2} b \pi+\frac{1}{4} \pi=\frac{2 n+1}{2} \pi,
$$

and so, after some algebra, we obtain the following approximate expression for the eigenvalues, valid wherever the asymptotic [Eq. (10)] is valid:

$$
E_{\alpha}=\frac{\omega_{s}^{2}}{\mu}\left(n+\frac{|m|}{2}+\frac{3}{4}\right)^{2}-\frac{\omega_{c} m}{2} .
$$

A similar energy spectrum may be derived from the BohrSommerfeld quantization conditions,

$$
E=\frac{\omega_{s}^{2}}{\mu}\left(n+\frac{\tilde{m}+1}{2}\right)^{2}-\frac{\omega_{c} m}{2},
$$

where $\tilde{m}=\sqrt{m^{2}-1 / 4}$.

Up to a phase factor, this is very similar to Eq. (12), which is derived from the asymptotic form of the confluent hypergeometric function. In what follows, we shall tend to use the spectrum [Eq. (12)] unless otherwise stated since there is a possibility that the Bohr-Sommerfeld conditions do not work as well as they might, as has been noted in the case of a nanowire in a transverse electric field (as observed in Ref. 22, for example).

\section{B. Connecting the approximations with the parabolic confinement model}

We now turn to address what, if anything, Eqs. (12) and (13) have to do with the confinement potential spectrum ${ }^{8,10,19}$ (here, we take $\omega_{0}$ to be the frequency of the confining potential used in those sources). For simplicity, we shall set $\omega_{c}$ $=0$ (i.e., work in the limit of zero magnetic field). Defining $\xi=E-\mu$ and working where $E \approx \mu$, we may write the following for our nonlinear spectra [Eqs. (12) and (13)]:

$$
\xi=\frac{\omega_{s}^{2}}{\mu} \mathcal{A}^{2}-\mu \approx 2 \omega_{s} \mathcal{A}-2 \mu,
$$

where

$$
\mathcal{A}=\left\{\begin{array}{lr}
\left(n+\frac{|m|}{2}+\frac{3}{4}\right) & \text { asymptotic } \\
\left(n+\frac{\tilde{m}+1}{2}\right) & \text { semiclassical. }
\end{array}\right.
$$

When $\omega_{c}=0$ and $E \approx \mu$, the spectrum of the parabolic confinement model becomes

$$
\xi=E-\mu=2 \omega_{0}\left(n+\frac{|m|+1}{2}\right)-\mu .
$$

So, in this limit, the confinement model, if we take $\omega_{0}$ $\equiv \omega_{s}$, and the nonlinear approximations are good matches up to an unimportant phase and a factor of 2 in front of the $\mu$ term. Since the latter only shifts the value of the energy by a 
constant, it seems that the results of Ref. 19 are a good approximation of the behavior of the nonlinear approximations [Eqs. (12) and (13)], very near the Fermi energy and at zero field.

This motivates a replacement of $\omega_{s}$ in Eq. (14) and, hence, in Eqs. (12) and (13), with $\omega$ so as to better approximate the behavior of the system at larger values of $\omega_{c}$ with these nonlinear spectra. That this is not a bad approximation (at least for small and intermediate values of $m$ ) is suggested by the numerical analysis of Sec. IV.

Having made this replacement and restoring the momentum component in the $z$ direction, we may write the spectra of the nonlinear model [Eqs. (12) and (13)] of electrons confined in a nanowire as

$$
E=\frac{k^{2}}{2}+\frac{\omega^{2}}{\mu} \mathcal{A}^{2}-\frac{\omega_{c} m}{2} .
$$

It is also instructive to compare this with the spectrum used by Bogachek and Gogadze ${ }^{13}$ and Dingle. ${ }^{12}$ Perturbation theory for small values of the field gives us the following expression for the energy:

$$
E=\frac{\epsilon_{n m}}{2 m^{*} R^{2}}+\frac{k^{2}}{2 m^{*}},
$$

where in this case we restore $m^{*}$ for clarity, and

$$
\epsilon_{n m}=\gamma_{n m}^{2}-2 \phi m+\frac{1}{3} \phi^{2}\left[1+\frac{2\left(m^{2}-1\right)}{\gamma_{n m}^{2}}\right],
$$

with $\gamma_{n m}$ being the $n$th zero of the $m$ th order Bessel function, and $\phi=\Phi / \Phi_{0}$, where $\Phi=\pi R^{2} B$ and $\Phi_{0}=h c / e$ (in ordinary units). Defining a frequency $\nu_{s}=v_{F} / R$, our overall expression for the energy becomes

$$
E=\frac{k^{2}}{2}+\frac{\nu_{s}^{2}}{4 \mu} \gamma_{n m}^{2}-\frac{\omega_{c}}{2}+\frac{\phi \omega_{c}}{12}\left[1+\frac{2\left(m^{2}-1\right)}{\gamma_{n m}^{2}}\right] .
$$

Discarding the last term and noting that the zeros of the Bessel function in our region of interest (as opposed to those of Bogacheck and Gogadze ${ }^{13}$ and Dingle ${ }^{12}$ ) are given by $\pi\left(n+\frac{|m|}{2}+\frac{3}{4}\right)$, we arrive at our asymptotic expression for the energy at a weak field. It is possible to recover the AharanovBohm oscillations predicted by Bogacheck and Gogadze from the results of our calculations in the next section by examining the limit of small $\omega_{c}$. However, the oscillations they obtain have the form $\cos (2 \pi r \phi)$, whereas ours have the form $\cos (4 r \phi / \pi)$. It seems probable that for the most part this discrepancy is due to our use of a different asymptotic approximation of the Bessel function zeros-they use an approximation valid for large $m$ and we use one valid for large $n$.

\section{C. de Haas-van Alphen effect for the nonlinear model}

We now calculate the oscillatory portion of the thermodynamic potential for the asymptotic version of Eq. (17) henceforth referred to as the nonlinear model-neglecting the phase term in the quadratic portion since it is much smaller than $n+\frac{|m|}{2}$, so that we have

$$
E_{n m k}=\frac{k^{2}}{2}-\frac{m \omega_{c}}{2}+\frac{\omega^{2}}{\mu}\left(n+\frac{|m|}{2}\right)^{2} .
$$

Applying twice Poisson's formula to the expression for the thermodynamic potential

$$
\Omega=-T \sum_{\alpha} \ln \left[1+e^{\left(\mu-E_{\alpha}\right) / T}\right],
$$

and replacing negative $m$ with $-(m+1)$, one obtains

$$
\Omega=\sum_{r, r^{\prime}=-\infty}^{\infty}\left[\Omega_{r r^{\prime}}^{+}+\Omega_{r r^{\prime}}^{-}\right]
$$

where

$$
\begin{aligned}
\Omega_{r r^{\prime}}^{ \pm}= & -\frac{2^{5 / 2} T \mu^{5 / 2}}{\pi \omega^{2}} \int_{0}^{\infty} d k \int_{0}^{\infty} d x \int_{0}^{\infty} d y e^{i(p x+q y)} \\
& \times \ln \left[1+e^{\mu\left[1-(x+y)^{2}-k^{2} \pm \beta y\right] / T}\right],
\end{aligned}
$$

with $p=2 \pi \mu r / \omega, q=4 \pi \mu r^{\prime} / \omega$, and $\beta=\omega_{c} / \omega$. We are interested in the part of $\Omega$ oscillating with $B$, which arises from terms in Eq. (4) with nonzero $r, r^{\prime}$. Replacing $(x+y)$ for $z$ and introducing the polar-spherical coordinates yield

$$
\begin{aligned}
\widetilde{\Omega}_{r r^{\prime}}^{ \pm}= & -\frac{2^{5 / 2} T \mu^{5 / 2}}{\pi \omega^{2}} \int_{0}^{\infty} d y e^{i(q-p) y} \int_{0}^{\pi / 2} d \phi \\
& \times \int_{y / \cos \phi}^{\infty} d \rho \rho e^{i p \rho \cos \phi} \ln \left[1+e^{\mu\left(1-\rho^{2} \pm \beta y\right) / T}\right] .
\end{aligned}
$$

Replacing $y$ for $y=\left(\rho^{2}-\epsilon\right) /( \pm \beta)$, we obtain

$$
\begin{aligned}
\Omega_{r r^{\prime}}^{+}= & -\frac{2^{5 / 2} T \mu^{5 / 2}}{\pi \omega^{2} \beta} \int_{0}^{\infty} d \epsilon e^{i(p-q) \epsilon / \beta} \ln \left[1+e^{\mu(1-\epsilon) / T}\right] \\
& \times \int_{0}^{\pi / 2} d \phi \int_{\sqrt{\epsilon}}^{\gamma(\epsilon, \phi)} d \rho \rho e^{i p \rho \cos \phi+i(q-p) \rho^{2} / \beta},
\end{aligned}
$$

where $\gamma(\epsilon, \phi)=\left[\epsilon+\beta^{2} \cos ^{2}(\phi) / 4\right]^{1 / 2}+\beta \cos (\phi) / 2 . \Omega_{r r^{\prime}}^{-}$is obtained by replacing $\beta$ for $-\beta$ in this expression. Here, we neglect a contribution from regions where the total energy $\epsilon$ is negative. This contribution is an artifact of approximation (21), and, in any case, it is exponentially small at temperatures $T \ll \mu$ in the oscillating part of $\Omega$.

Neglecting terms of the order of $\omega / \mu \ll 1$, the integral over $\rho$ is approximated as

$$
\begin{aligned}
I \equiv & \int_{\sqrt{\epsilon}}^{\gamma(\epsilon, \phi)} d \rho \rho e^{i p \rho \cos \phi+i(q-p) \rho^{2} / \beta} \\
\approx & i \beta\left[\frac{\sqrt{\epsilon} e^{i p \sqrt{\epsilon} \cos \phi+i(q-p) \epsilon / \beta}}{p \beta \cos \phi+2(q-p) \epsilon}\right. \\
& \left.-\frac{\gamma(\epsilon, \phi) e^{i p \gamma(\epsilon, \phi) \cos \phi+i(q-p) \gamma^{2}(\epsilon, \phi) / \beta}}{p \beta \cos \phi+2(q-p) \gamma(\epsilon, \phi)}\right] .
\end{aligned}
$$

At low temperatures, $T \ll \mu$, the main contribution to the oscillating part of the thermodynamic potential $\widetilde{\Omega}$ comes from energies near the Fermi surface, $|1-\epsilon| \ll 1$. Moreover at large $p \gg 1$, only small angles $(\phi \ll 1)$ contribute to the inte- 
gral, which allows us to extend the integration over $\phi$ up to infinity. We can also replace $\cos (\phi)$ by $1-\phi^{2} / 2$, expand $\gamma(\epsilon, \phi)$ as

$$
\gamma(\epsilon, \phi) \approx \gamma-\frac{|1-\epsilon|}{2(\gamma-\beta / 2)}-\frac{\beta \gamma \phi^{2}}{4[\gamma-\beta / 2]},
$$

in the exponents (here $\gamma=\sqrt{1+\beta^{2} / 4}+\beta / 2$ ), and take $\cos \phi$ $=1, \gamma(\epsilon, \phi)=\gamma$ in the pre-exponential terms. Integrating over $\phi$ and $|1-\epsilon|$ with the use of $\int_{0}^{\infty} d \phi \exp \left(i a \phi^{2}\right)$ $=(\pi / 4|a|)^{1 / 2} \exp [i \pi a /(4|a|)] \quad$ and $\quad \int d x \exp ($ iax $)[1$ $+\exp (x)]^{-1}=-i \pi / \sinh (\pi a)$ yields

$$
\begin{aligned}
\widetilde{\Omega}_{r r^{\prime}}^{+}= & i \frac{8 \pi^{1 / 2} T \mu^{5 / 2}}{\omega^{2}}\left[\frac{e^{i p-i \pi p / 4|p|}}{p|p|^{1 / 2} \sinh (\pi p T / 2 \mu)[p \beta+2(q-p)]}\right. \\
& \left.-\frac{(\gamma-\beta / 2)^{3 / 2} e^{i q \gamma-i \pi q / 4|q|}}{q|q|^{1 / 2} \sinh [\pi q T / 2 \mu(\gamma-\beta / 2)][p \beta+2(q-p) \gamma]}\right]
\end{aligned}
$$

This approximation fails at the magic resonances, where $p \beta+2(q-p)=0$ or $p \beta+2(q-p) \gamma=0$. In those cases, the integral $[$ Eq. (27)] is calculated as

$$
I \approx(\pi / 4|p|)^{1 / 2} e^{i p \sqrt{\epsilon} \cos \phi+i(q-p) \epsilon / \beta-i \pi p / 4|p|}
$$

or

$$
I \approx \gamma[\pi(\gamma-\beta / 2) / 4|q|]^{1 / 2} e^{i p \gamma(\epsilon, \phi) \cos \phi+i(q-p) \gamma^{2}(\epsilon, \phi) / \beta-i \pi q / 4|q|},
$$

respectively. Combining Eq. (28) and Eqs. (30) and (31), we can replace the resonant denominators in Eq. (27) as

$\frac{1}{p \beta+2(q-p)} \Rightarrow \frac{1}{p \beta+2(q-p)+i \beta(4|p| / \pi)^{1 / 2} \exp (i \pi p / 4|p|)}$

and

$$
\begin{aligned}
& \frac{1}{p \beta+2(q-p) \gamma} \\
& \quad \Rightarrow \frac{1}{p \beta+2(q-p) \gamma-i \beta[4|q| / \pi(\gamma-\beta / 2)]^{1 / 2} \exp (i \pi q / 4|q|)} .
\end{aligned}
$$

Substituting this expression into Eq. (23) and performing partial summation yields

$$
\begin{aligned}
\widetilde{\Omega}= & \sum_{r=1}^{\infty} \sum_{ \pm} A_{r}^{ \pm} \sin (2 \pi r \mu / \omega-\pi / 4)+B_{r}^{ \pm} \cos (2 \pi r \mu / \omega-\pi / 4) \\
& +C_{r}^{ \pm} \sin \left[4 \pi r \mu / \widetilde{\omega}^{\mp}-\pi / 4\right]+D_{r}^{ \pm} \cos \left[4 \pi r \mu / \widetilde{\omega}^{\mp}-\pi / 4\right],
\end{aligned}
$$

where

$$
\begin{aligned}
A_{r}^{ \pm}= & \frac{T(2 \omega)^{1 / 2}}{2 \pi r^{3 / 2} \sinh \left(\pi^{2} T r / \omega\right)} \\
& \times \Re \cot \left[\pi r \omega^{\mp} / \omega \pm \beta(1-i)(r \omega / \mu)^{1 / 2} / 4\right],
\end{aligned}
$$

$$
\begin{aligned}
B_{r}^{ \pm}= & \frac{T(2 \omega)^{1 / 2}}{2 \pi r^{3 / 2} \sinh \left(\pi^{2} T r / \omega\right)} \\
& \times \mathfrak{I} \cot \left[\pi r \omega^{\mp} / \omega \pm \beta(1-i)(r \omega / \mu)^{1 / 2} / 4\right], \\
C_{r}^{ \pm}= & \frac{T\left(\widetilde{\omega}^{+}+\widetilde{\omega}^{-}\right)^{1 / 2}}{4 \pi r^{3 / 2} \sinh \left[4 \pi^{2} T r /\left(\widetilde{\omega}^{+}+\widetilde{\omega}^{-}\right)\right]} \\
& \times \Re \cot \left[2 \pi r \widetilde{\omega}^{ \pm} /\left(\widetilde{\omega}^{+}+\widetilde{\omega}^{-}\right)\right. \\
& \left. \pm \beta(1-i) \omega^{2}(r / \mu)^{1 / 2} / 2\left(\widetilde{\omega}^{+}+\widetilde{\omega}^{-}\right)^{3 / 2}\right],
\end{aligned}
$$

and

$$
\begin{aligned}
D_{r}^{ \pm}= & \frac{T\left(\widetilde{\omega}^{+}+\widetilde{\omega}^{-}\right)^{1 / 2}}{4 \pi r^{3 / 2} \sinh \left[4 \pi^{2} \operatorname{Tr} /\left(\widetilde{\omega}^{+}+\widetilde{\omega}^{-}\right)\right]} \\
& \times \Im \cot \left[2 \pi r \widetilde{\omega}^{ \pm} /\left(\widetilde{\omega}^{+}+\widetilde{\omega}^{-}\right)\right. \\
& \left. \pm \beta(1-i) \omega^{2}(r / \mu)^{1 / 2} / 2\left(\tilde{\omega}^{+}+\widetilde{\omega}^{-}\right)^{3 / 2}\right] .
\end{aligned}
$$

Here, $\omega^{ \pm}=\omega \pm \omega_{c} / 2, \widetilde{\omega}^{ \pm}=\omega\left(\sqrt{1+\beta^{2} / 4} \pm \beta / 2\right)$, and the summation formula, $\Sigma_{r}(z-r)^{-1}=\pi \cot (\pi z)$ has been applied. The \pm of $A_{r}^{ \pm}$cancel with each other, as do those of $B_{r}^{ \pm}$. This allows us to simplify our formula and write

$$
\begin{aligned}
\widetilde{\Omega}= & \sum_{r=1}^{\infty} \sum_{ \pm} C_{r}^{ \pm} \sin \left[4 \pi r \mu / \tilde{\omega}^{\mp}-\pi / 4\right] \\
& +D_{r}^{ \pm} \cos \left[4 \pi r \mu / \tilde{\omega}^{\mp}-\pi / 4\right] .
\end{aligned}
$$

We can see that, unlike the AK result, we have two characteristic fequencies $\widetilde{\omega}^{ \pm} / 2$ which differ from the equivelent frequencies in that result. In addition, the magic resonances occur at different ratios of $\omega_{c} / \omega_{s}$ and are damped by the additional terms in the cotangent function. As is usual in these calculations, we may restore the effects of spinsplitting by multiplying each term by $\cos \left(r \pi g \mu_{B} / e\right)$. It should be noted that the effects of spin orbital coupling and other complications may further complicate this expression in real materials.

\section{NUMERICAL RESULTS}

Here, we present some numerical calculations of the DHVA oscillations and of their Fourier transforms so that the $\mathrm{AK}$ and nonlinear results might be compared. We have set $\mu=2000 \omega_{s}$ and $\omega_{s}=1$, measuring $\omega_{c}$ and all other quantities in units of $\omega_{s}$. Fourier transfromations were performed using the NAG DFT routine C06FAF and the Fourier integral calculation techniques described in Ref. 23.

Calculations at $T=0$ for the parabolic confinement model were performed through taking the limit $T \rightarrow 0$ in Eq. (22) and then integrating over $k$. We thus obtained the following expression for the thermodynamic potential,

$$
\Omega=-\frac{4 \sqrt{2}}{3 \pi} \sum_{n, m} \mathfrak{R}\left(\mu-E_{n m}\right)^{3 / 2},
$$

where $E_{n m}$ is energy for a given $n, m$. The nonoscillatory portion was then subtracted out, leaving only the oscillatory portion of the function behind. 

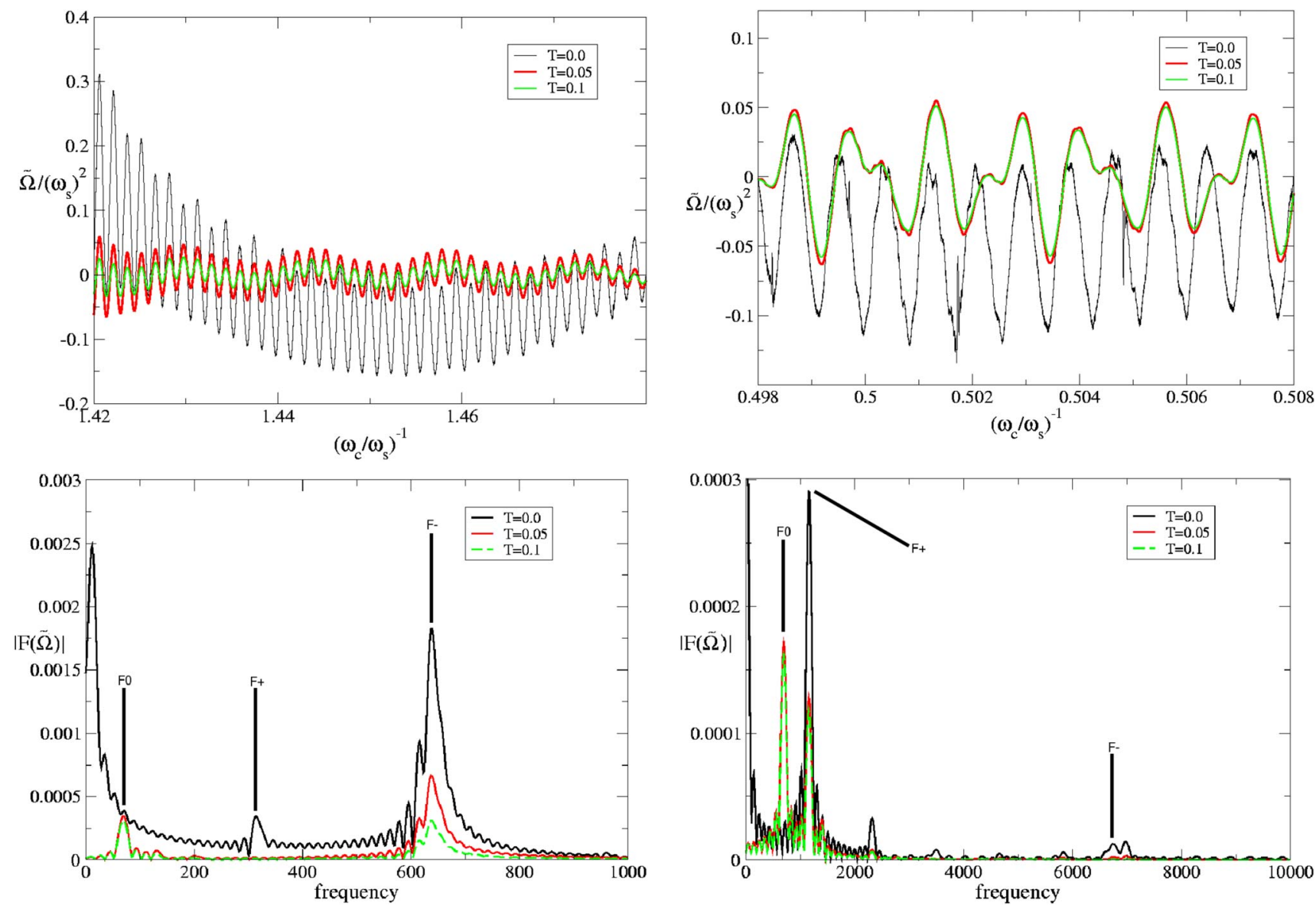

FIG. 1. (Color online) Graphs of $\widetilde{\Omega}$ in the parabolic confinement model above their Fourier transforms. Note that the remnant of the nonperiodic component of the $T=0$ data is most noticeable in the top left graph, but also that the $F+$ resonance is most obviously visible in its Fourier transform.

This could not be done in the case of the nonlinear model, however, due to the issue remarked on in Sec. II C regarding the unphysical negative energy levels. It appears that as yet there is no straightforward way of calculating the thermodynamic quantities in this fashion, as it does not appear as easy to remove the contributions from negative energy values in this method as it was in the aforementioned analytic (Poisson summation) treatment of Sec. II C. For $T>0$, the calculations were done using the formulas of Ref. 19 for the AK results, and Eqs. (39) and (38) in this paper were used for the nonlinear results.

Figure 1 displays the behavior of the thermodynamic potential with respect to changes in $\left(\omega_{c}\right)^{-1}$, alongside their Fourier transforms. These last clearly show the three peaks corresponding to each of the characteristic frequencies given in Ref. 19 (here, $F_{0}$ is equivalent to $F$ of that reference). In the case of the $T=0$ plots, it seems that some residual nonoscillatory components of the untransformed functions remain at low frequencies, and these obscure the low frequency content of the Fourier transform.

In Fig. 2, we present the first magic resonance in the thermodynamic potential of the nonlinear model together with that of the parabolic confinement model for the purposes of comparison. We can see that the resonance in the nonlinear case has not only changed position, but has also been damped by the additional terms in the cotangent functions of the Fourier coefficients.

Figure 3 shows the thermodynamic potential and its Fourier transform in the same ranges as previously used for the

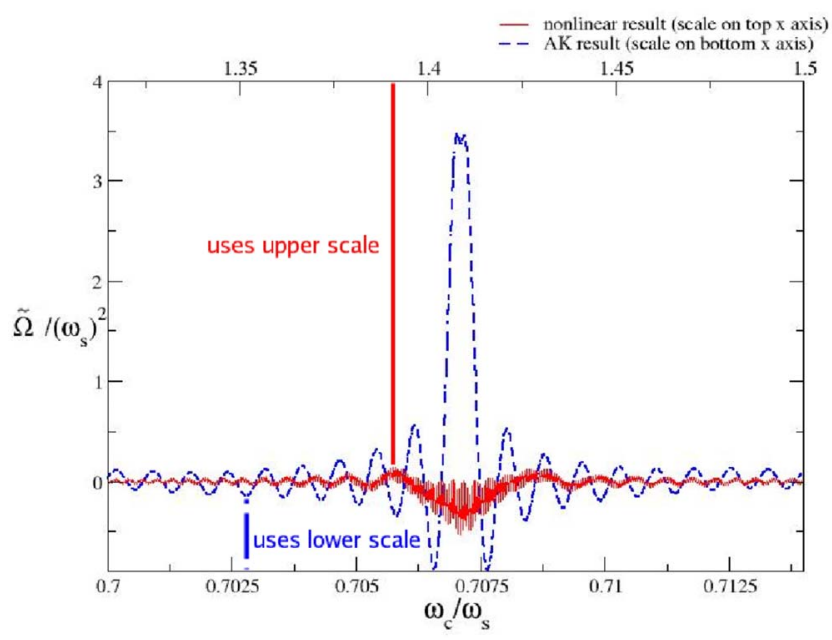

FIG. 2. (Color online) Graph comparing the first magic resonances of the parabolic confinement model with the nonlinear model. The temperature here is set at $T=0.025$. 

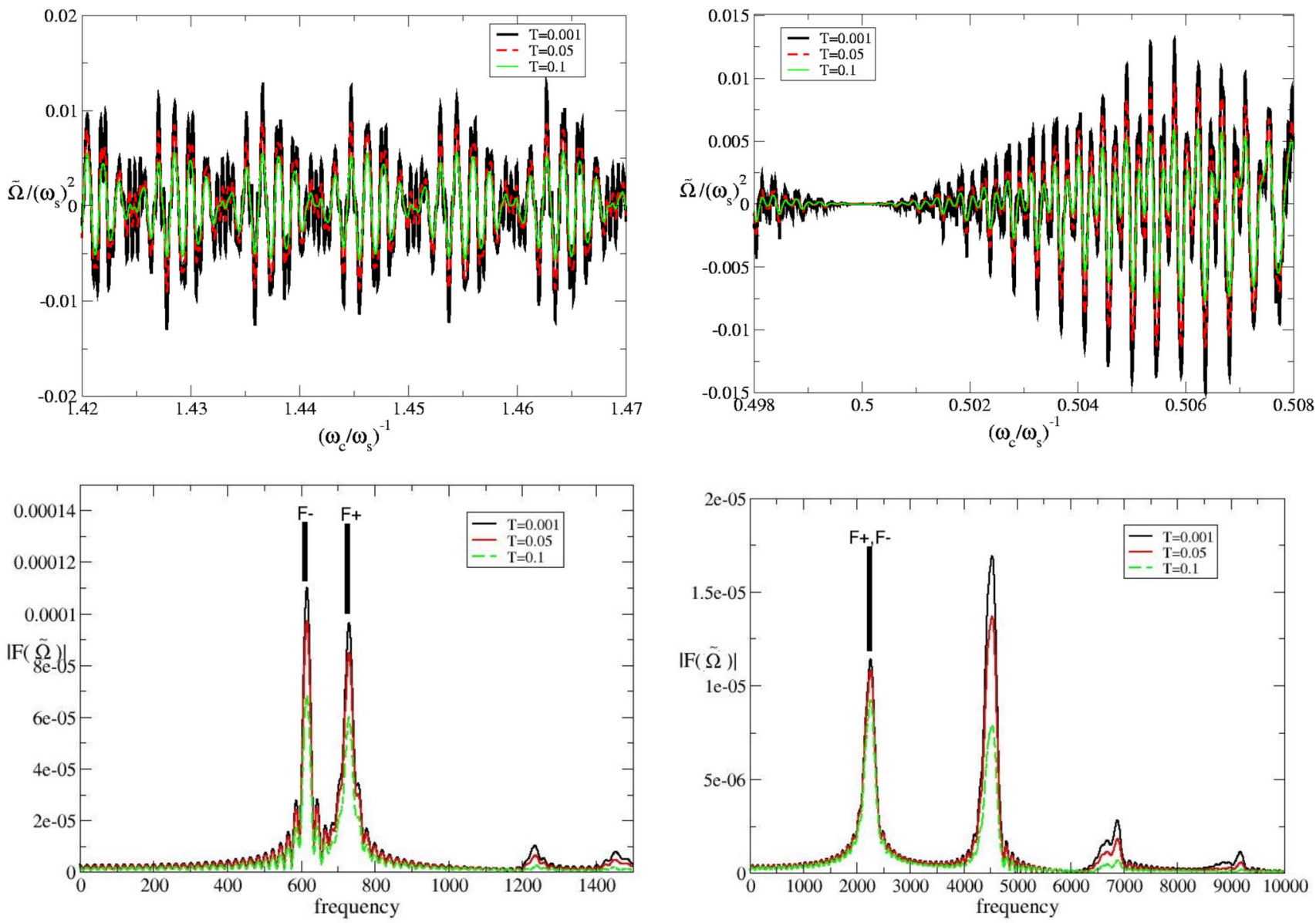

FIG. 3. (Color online) Graphs of $\widetilde{\Omega}$ in the nonlinear model above their Fourier transforms. Note that the $F+$ and $F-$ peaks in the lower left Fourier transform are so close as to be indistiguishable; the second harmonic is larger than expected here due to its enhancement by the cotangent portion of its amplitude.

parabolic confinement model calculations. Following Ref. 19, we may estimate the characteristic frequencies as being

$$
F^{ \pm}=\frac{2 F\left|\sqrt{2} \pm \sqrt{1+\gamma^{\prime}}\right|}{\sqrt{1+\gamma^{\prime}}\left[\sqrt{1+\gamma^{\prime}} \pm(1 / \sqrt{2})\right]^{2}},
$$

where $F=S_{F} /(2 \pi e), \quad \gamma=4 \omega_{s}^{2} / \omega_{c}^{2}=4 \pi^{2} S_{F} /\left(e^{2} S B^{2}\right), \quad \gamma^{\prime}=\gamma / 2$, $S=\pi R^{2}$ is the cross-sectional area of the wire, and $S_{F}$ $=\pi\left(m^{*}\right)^{2} v_{F}^{2}$ is the cross-sectional area of the Fermi surface. (We remind the reader that in order to interpret the frequencies given in the figures using these formulas, we should take $e=m^{*}=\omega_{s}=1$.)

We can see the two characteristic frequencies clearly in the Fourier transform, and that the oscillatory behavior of the function is considerably different from that seen in the parabolic confinement case.

\section{HOW GOOD ARE THE APPROXIMATIONS?}

In order to understand how our nonlinear model differs from the exact behavior of the system, we must compare their spectra with that generated from the exact zeros of the confluent hypergeometric function $M(a, b, \xi)$. In order to ob- tain the latter, we numerically generated $M(a, b, \xi)$ with the CONHYP function ${ }^{24,25}$ and located the roots of the function as the parameter $-a$ was increased from zero using the van Wijngaarden-Dekker-Brent algorithm. ${ }^{26}$

Unfortunately, the processing ability of the computer limits the values of the variable $\xi$ for which results can be obtained; this means that it is difficult to obtain zeros for large values of both $\mu / \omega_{s}$ and $\omega_{c} / \omega_{s}$ since this entails a large value of $\xi$ that will cause the series defining $M(a, b, \xi)$ to be very slowly converging. However, one can gain an idea of the behavior of the exact system for less extreme values of the variable, and it is to such an example that we now turn.

We set $\mu=100 \omega_{s}, \omega_{s}=1$, and examine values of 0 $\leqslant \omega_{c} / \omega_{s} \leqslant 1$. For each of these, we determine the largest value of $n$ in the nonlinear approximation for which $E \leqslant \mu$ (assuming $k$ is zero) and calculate the relative error $\Delta=\left(E-E_{\text {exact }}\right) / E$, where $E_{\text {exact }}$ is the exact energy calculated from the $n$th zero of $M(a, b, \xi)$. This enables us to gain some idea of the size of the error at the Fermi surface described by the nonlinear model.

To obtain the exact energy in the zero-field limit, we note that the wave function in that case takes the form of a Bessel function, and so the exact zeros may be obtained from the function $J_{|m|}\left(2 \sqrt{E_{\text {exact }} w}\right)$, where $w=\left(\frac{\pi}{2 \omega_{s}}\right)^{2} \mu$. 


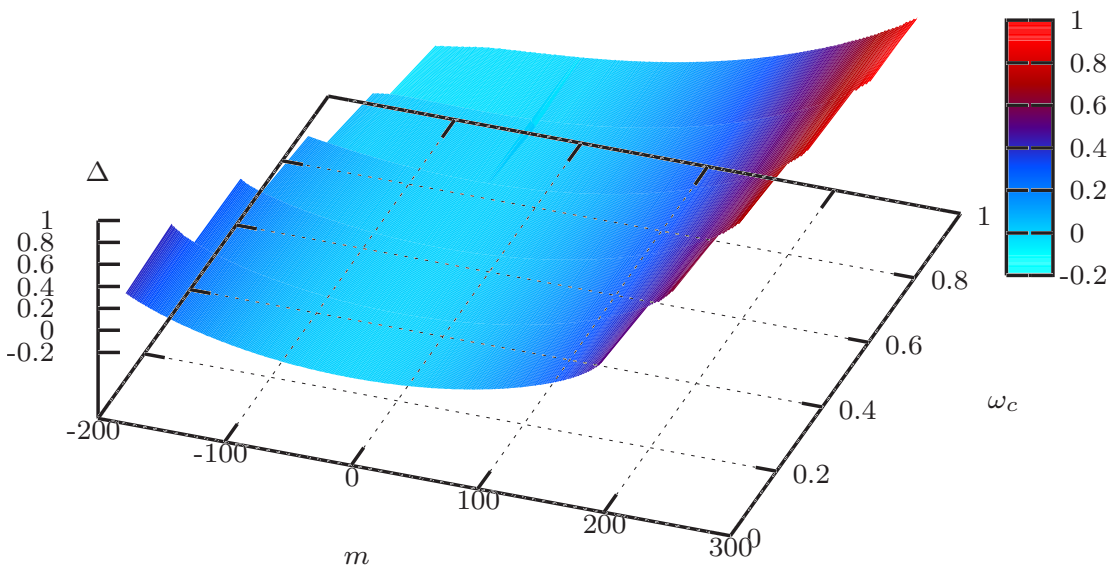

FIG. 4. (Color online) The relative error $\Delta$ along the Fermi surface defined by the nonlinear model, as a function of $m$ and $\omega_{c}$.
We plot $\Delta$ in Fig. 4. It is apparent that as $\omega_{c}$ increases, the shape of the approximate Fermi surface alters such that the maximum possible positive value of $m$ increases and the maximum negative value of $m$ decreases. This coincides with a decrease in the maximum value of $|\Delta|$ for negative $m$ and an increase for positive $m$. In fact, the latter error is so large for large $m$ at $\omega_{c}=1$ that it can scarcely be said that it accurately models the exact values at all in that region. In addition, it is worth noting that the nonlinear model underestimates the deformation of the Fermi surface when $\omega_{c}$ is large (for example, see Fig. 5). Why is this?

In the case of the asymptotic derivation of the model, the asymptotic from which we obtained it makes two assumptions: that $-a$ is greater than $\xi$, so that $M(a, b, \xi)$ resembles a Bessel function, and that $-a$ is greater than $b$, so that the Bessel function resembles a cosine function. Obviously, this condition is violated in the zero-field limit at large $m$, and so we would expect it to be violated for large $m$ at finite values of the field, even though it appears that the replacement of $\omega_{s}$ with $\omega$ in Sec. II B counteracts the violation of the first condition for large $\xi$ if $b$ is small. This is what occurs in the case of positive $m$. As for the the portions of the spectrum with negative $m$, since these have larger energies than their positive counterparts and since this disparity increases as we increase $\omega_{c}$, one should not be surprised to find that as $\omega_{c}$

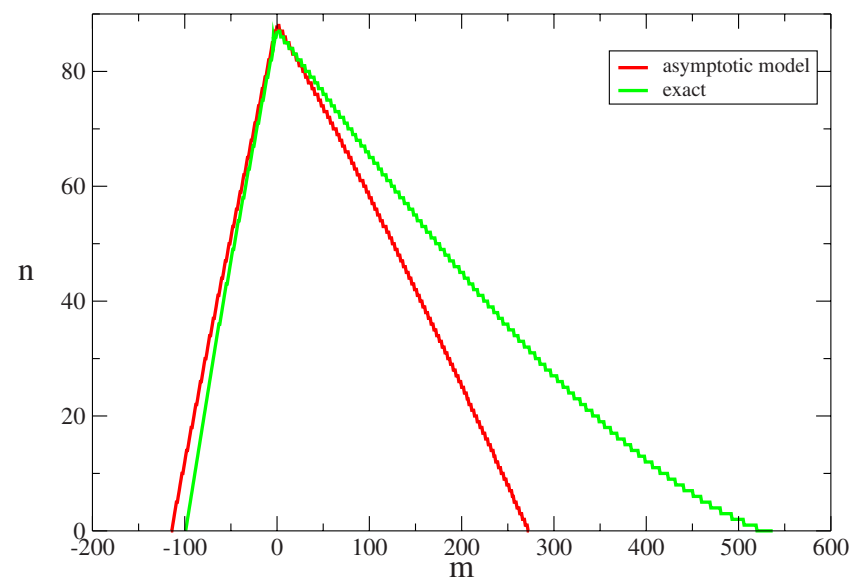

FIG. 5. (Color online) Graph showing the quantum number $n$ against $m$ at the Fermi surface for $\mu=100 \omega_{s}$ and $\omega_{c} / \omega_{s}=1.0$. increases, the Fermi energy is exceeded at smaller and smaller values of negative $m$, which are in turn more closely matched by the asymptotic. So, in this case, the maximum error decreases.

Semiclassically, one should note that large values of $m$ describe electron orbits that approach the edge of the wire quite closely. Since that is a turning point due to the imposition of the hard boundary conditions, we cannot expect the semiclassical approximation to hold in that region. The analysis of the difference between the behavior at positive and negative $m$ given above also holds here.

Perhaps we should consider the boundary conditions of our problem. The parabolic confinement model was originally derived with "soft" boundary conditions - a parabolic confining potential-and as we have shown, this approximates the nonlinear model, derived with hard boundary conditions near the Fermi surface. However, this latter model does not capture the large $m$ behavior of the system very well, in part, one suspects, because of the hardness of the boundary conditions. It should be noted then that neither of these extremes is likely to be physical-the boundary conditions for a real nanowire will most probably be intermediate between them, and so it is possible that the experimental behavior of the system might also be intermediate between the behaviors we have discussed. This, of course, is a matter that requires empirical determination.

\section{CONCLUSIONS}

We have calculated the oscillations in the thermodynamic potential due to the de Haas-van Alphen effect in a clean metallic nanowire with a simple Fermi surface for a nonlinear energy spectrum derived from an asymptotic approximation to the exact solution of the Schrödinger equation, and have compared them with the AK results derived using the parabolic confinement model which approximates it near the Fermi energy. In both models, one can observe magic resonances where the amplitude of the oscillations in the thermodynamic potential is enhanced at particular ratios of $\omega_{s}$ to $\omega_{c}$, although their locations differ in each model and are somewhat damped in the nonlinear case. However, the nonlinear result lacks one of the frequencies of oscillation observed in the $\mathrm{AK}$ result. In addition, it seems that the infinite well boundary conditions cause some problems at large values of 
$m$ due to the semiclassical nature of our approximations; however, this is unlikely to be fatal to our predictions of magic resonances since the real system likely has boundary conditions intermediate between the two cases which we have considered.

It seems clear that the interplay between the size quantization and the magnetic quantization is nontrivial in the extreme and exhibits a certain degree of model dependence. Further experimental and theoretical work is needed both to clarify remaining ambiguities and to apply this general theory to the more complex band structures of realistic nanowires.

\section{ACKNOWLEDGMENTS}

The authors would like to thank the EPSRC for funding this research (Grant No. EP/D035589) and David Khmelnitskii for helpful comments.
${ }^{1}$ W. J. de Haas and P. M. van Alphen, Proceedings of the Netherlands Royal Academy of Sciences 33, 680 (1930); 33, 1106 (1930); 35, 454 (1932).

${ }^{2}$ L. Onsager, Philos. Mag. 43, 1006 (1952).

${ }^{3}$ I. M. Lifshitz and A. M. Kosevitch, Zh. Eksp. Teor. Fiz. 29, 730 (1955) [Sov. Phys. JETP 2, 636 (1956)].

${ }^{4}$ A. V. Gold, Solid State Physics: The Simon Fraser University Lectures (Gordon and Breach, New York, 1968), Vol. 1, p. 39.

${ }^{5}$ D. Schoenberg, Magnetic Oscillations in Metals (Cambridge University Press, Cambridge, 1984).

${ }^{6}$ J. Singleton, Rep. Prog. Phys. 63, 1111 (2000).

${ }^{7}$ M. V. Kartsovnik, Chem. Rev. (Washington, D.C.) 104, 5737 (2004).

${ }^{8}$ V. Fock, Z. Phys. 47, 446 (1928).

${ }^{9}$ L. D. Landau, Z. Phys. 64, 629 (1930).

${ }^{10}$ C. G. Darwin, Proc. Cambridge Philos. Soc. 27, 86 (1930).

${ }^{11}$ R. B. Dingle, Proc. R. Soc. London, Ser. A 211, 500 (1952).

${ }^{12}$ R. B. Dingle, Proc. R. Soc. London, Ser. A 212, 47 (1952).

${ }^{13}$ E. N. Bogachek and G. A. Gogadze, Zh. Eksp. Teor. Fiz. 63, 1839 (1972) [Sov. Phys. JETP 36, 973 (1973)].

${ }^{14}$ N. B. Brandt, D. B. Gitsu, V. A. Dolma, and Ya. G. Ponomarev, Zh. Eksp. Teor. Fiz. 92, 913 (1987) [Sov. Phys. JETP 65, 515 (1987)].

${ }^{15}$ T. E. Huber, K. Celestine, and M. J. Graf, Phys. Rev. B 67, 245317 (2003); T. E. Huber, A. Nikolaeva, D. Gitsu, L. Konopko, C. A. Foss, Jr., and M. J. Graf, Appl. Phys. Lett. 84,
1326 (2004); A. Nikolaeva, D. Gitsu, L. Konopko, M. J. Graf, and T. E. Huber, arXiv:cond-mat/0702368 (unpublished).

${ }^{16}$ A. Fujiwara, K. Tomiyama, H. Suematsu, M. Yumura, and K. Uchida, Phys. Rev. B 60, 13492 (1999).

${ }^{17}$ S. Roche, G. Dresselhaus, M. S. Dresselhaus, and R. Saito, Phys. Rev. B 62, 16092 (2000).

${ }^{18}$ E. P. Sineavsky, R. A. Khamidullin, T. E. Huber, A. A. Nikolaeva, and L. A. Konopko, Rev. Adv. Mater. Sci. 8, 170 (2004).

${ }^{19}$ A. S. Alexandrov and V. V. Kabanov, Phys. Rev. Lett. 95, 076601 (2005).

${ }^{20}$ L. D. Landau and E. M. Lifshitz, Quantum Mechanics (NonRelativistic Theory), 3rd ed. (Pergamon, Oxford, 1977).

${ }^{21}$ Handbook of Mathematical Functions, edited by M. Abramowitz and I. A. Stegun (Dover, New York, 1972).

${ }^{22}$ S. Chaudhuri and S. Bandyopadhyay, J. Appl. Phys. 71, 3027 (1997).

${ }^{23}$ W. H. Press, S. A. Teukolsky, W. T. Vetterling, and B. P. Flannery, Numerical Recipies in FORTRAN, 2nd ed. (Cambridge University Press, Cambridge, 1992), pp. 577-584.

${ }^{24}$ M. Nardin, W. F. Perger, and A. Bhalla, ACM Trans. Math. Softw. 18, 345 (1992).

${ }^{25}$ M. Nardin, W. F. Perger, and A. Bhalla, J. Comput. Appl. Math. 39, 193 (1992).

${ }^{26}$ W. H. Press, S. A. Teukolsky, W. T. Vetterling, and B. P. Flannery, Numerical Recipies in FORTRAN (Ref. 23), pp. 352-355. 